observed between daily symptoms and daily assessments of activity function items $(r=0.66-0.77)$, feeling tired $(r=0.66-$ 0.76), and ACQ-6 scores $(\mathrm{r}=0.58-0.68)$ at baseline and change from baseline to end of treatment. Moderate correlations were observed between symptom scores and feeling stressed $(r=0.36-0.50)$; rescue medication use $(r=0.49-0.57)$; and AQLQ(S)+12 overall and symptoms, activity limitation, and emotional domains $(\mathrm{r}=-0.41-0.62)$. Although a very weak correlation between symptom improvement and $\mathrm{FEV}_{1}$ was expected, benralizumab-treated patients had a better correlation between symptom improvement and $\mathrm{FEV}_{1}$ improvement compared with placebo ( $\mathrm{r}=-0.21$ vs. -0.13$)$, possibly because of substantial $\mathrm{FEV}_{1}$ improvement observed in benralizumabtreated patients.

Conclusions Asthma-related symptoms and improvements are associated with other important aspects of improvement in patient well-being, especially for patients uncontrolled on optimal care.

\section{P69 FUNCTIONALITY, RELIABILITY, AND PERFORMANCE OF AN ACCESSORISED PRE-FILLED SYRINGE WITH HOME- ADMINISTERED SUBCUTANEOUS BENRALIZUMAB FOR ADULT PATIENTS WITH SEVERE ASTHMA}

${ }^{1} \mathrm{AH}$ Mansur, ${ }^{2} \mathrm{GT}$ Ferguson, ${ }^{3} \mathrm{JS}$ Jacobs, ${ }^{4} \mathrm{~J}$ Hebert, ${ }^{5} \mathrm{C}$ Clawson, ${ }^{6} \mathrm{~W}$ Tao, ${ }^{6} \mathrm{Y}$ Wu ${ }^{6} \mathrm{M}$ Goldman, on behalf of the GREGALE study investigators. ${ }^{1}$ Birmingham Heartlands Hospital, Birmingham, UK; ${ }^{2}$ Pulmonary Research Institute of Southeast Michigan, Farmington Hills, MI, US: ${ }^{3}$ Allergy and Asthma Clinical Research, Walnut Creek, CA, US: ${ }^{4}$ Centre de Recherche Appliqué en Allergie de Québec, Québec, QC, Canada; ${ }^{5}$ Medlmmune LLC, Gaithersburg, MD, US; ${ }^{6}$ AstraZeneca, Gaithersburg, MD, US

\subsection{6/thoraxjnl-2017-210983.211}

Introduction and Objectives Benralizumab, a humanised antieosinophil monoclonal antibody, is in development as an addon treatment for severe, uncontrolled, eosinophilic asthma. During Phase III trials, benralizumab significantly reduced annual asthma exacerbation rates and was well-tolerated. ${ }^{1,2}$ The GREGALE study (NCT02417961) assessed patient and caregiver-reported functionality, performance, and reliability of an accessorised pre-filled syringe (APFS) used to administer benralizumab subcutaneously in an at-home setting.

Methods Patients $(n=116)$ with severe, uncontrolled asthma despite receiving medium- or high-dosage inhaled corticosteroids and long-acting $\beta_{2}$-agonists, received up to five APFSadministered subcutaneous doses (Weeks 0, 4, 8, 12, and 16) of $30 \mathrm{mg}$ benralizumab. The first three doses were administered at the study sites. The patient/caregiver administered the last two doses at home. Endpoints included the percentage of patients/caregivers who successfully administered benralizumab at home, percentage of APFS returned to study sites and evaluated as functional, percentage of APFS returned as malfunctioning to Product Complaints, efficacy (Asthma Control Questionnaire 6 [ACQ-6]), safety, and pharmacodynamics (blood eosinophil count).

Results Nearly all patients and caregivers successfully administered benralizumab with an APFS at home (Week 12: 112/114, 98\%; Week 16: 108/109, 99\%; figure 1). Two at-home administrations were unsuccessful because of patient-use error. One APFS was recorded as nonfunctional because it was not returned for evaluation. Product Complaints identified only 1 APFS malfunction of 573 dispensed. Mean ACQ-6 scores decreased from baseline through all postbaseline time points through end of treatment (baseline: mean 2.14 [standard deviation $\{$ SD $\}$ 0.81]; Week 20: mean
1.40 [SD 0.90]). Near-complete depletion of eosinophils was observed at end of treatment vs. baseline (baseline: median 250 cells $/ \mu \mathrm{L}$ [interquartile range $\{\mathrm{IQR}\} 175-430 \mathrm{cells} / \mu \mathrm{L}]$; and Week 20: median 0 cells $/ \mu \mathrm{L}$ [IQR $0-10$ cells $/ \mu \mathrm{L}])$. Incidence of adverse events leading to benralizumab discontinuation was $2.6 \%$. Most common adverse events ( $\geq 5 \%$ of patients) were nasopharyngitis, upper respiratory tract infection, headache, and sinusitis. Five patients $(4.3 \%)$ experienced transient mild or moderate injectionsite reactions.

Conclusions Most patients and caregivers successfully administered benralizumab in an at-home setting. The APFS was functional, reliable, and performed well.

Please refer to page A258 for declarations of interest in relation to abstract P69.

\section{REFERENCES}

1. Bleecker ER et al. Lancet 2016;388:2115-27.

2. FitzGerald JM et al. Lancet 2016:388:2128-41.

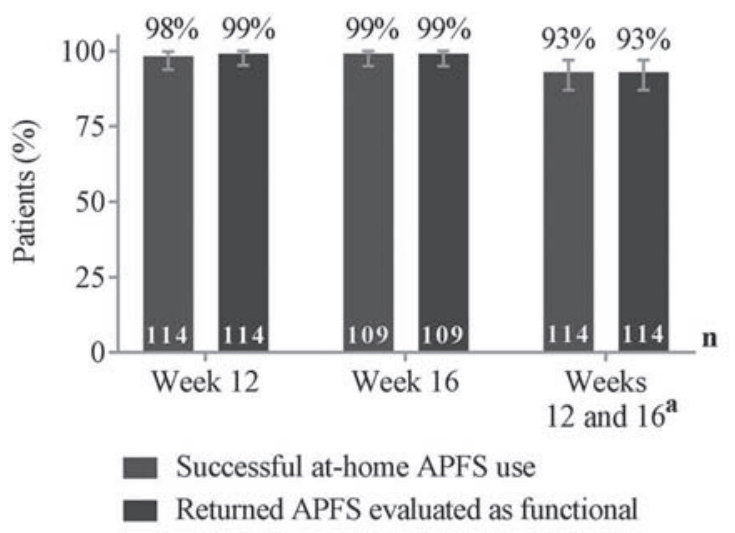

APFS, accessorized pre-filled syringes.

${ }^{a}$ Includes patients who were qualified for home-administration and were still in the study at Week 12 .

Error bars represent $95 \%$ confidence intervals calculated using the Clopper-Pearson exact method.

Abstract P69 Figure 1 Percentage of patients and caregivers who used an APFS successfully at home and returned APFS evaluated as functional.

\section{P70 RELATION BETWEEN BRONCHIAL ASTHMA AND PARASITIC (NEMATODES) INFECTION IN EGYPTIAN CHILDREN}

${ }^{1}$ YM Bakr, ${ }^{2} \mathrm{MM}$ Shahin, ${ }^{2} \mathrm{MH}$ Zidan, ${ }^{3} \mathrm{AA}$ Alfatah, ${ }^{2}$ JS Gharraf. ${ }^{1}$ Alexandria Police Hospital, Alexandria, Egypt; ${ }^{2}$ Alexandria Medicine Faculty, Alexandria, Egypt; ${ }^{3}$ Alexandria Medical High Institute, Alexandria, Egypt

\subsection{6/thoraxjnl-2017-210983.212}

Background Among the many factors influencing the prevalence of asthma in developing countries from the tropics are geo-helminthic infections.

Aims This work aims to study the relation between bronchial asthma and parasitic infestation in Egyptian children.

Patients and Methods A cross-section, analytical study design was chosen to perform this research on 100 school aged children. All children were interviewed and examined clinically and laboratory. 